\title{
6-烷氨基-2-乙硫基嘌呤核苷化合物的合成及抗血小板聚集活性研究
}

\author{
王子健 $^{a}$ 李顺来 ${ }^{a}$ 于明武 ${ }^{a}$ 骆 媛 ${ }^{b}$ 王 帅 ${ }^{b}$ \\ 王永安 ${ }^{*}, b$ 杜洪光*,a \\ ( ${ }^{a}$ 北京化工大学理学院 北京 100029) \\ $(b$ 军事医学科学院毒物药物研究所 抗毒药物与毒理学研究国家重点实验室 北京 100850)
}

\begin{abstract}
摘要 以鸟苷(1)为原料, 与乙酸酐经过糖环羟基保护反应, 得到 $2^{\prime}, 3^{\prime}, 5^{\prime}$-三- $O$-乙酰基鸟苷 $(2) ; 2$ 与对甲苯磺酰氯反应, 得到 9-(2',3',5'-三- $O$-乙酰基- $\beta$-D-呋喃核糖)-2-氨基-6-对甲苯磺酰氧基嘌呤 $(3) ; 3$ 与亚硝酸异戊酯和二乙基二硫醚反应, 得到 9-(2',3',5'-三- $O$-乙酰基- $\beta$-D-呋喃核糖)-6-对甲苯磺酰氧基-2-乙硫基嘌呤(4); 4 经过胺解和脱糖环羟基保护反应得到 10 个未见报道的 6-烷氨基-2-乙硫基嘌呤核苷化合物(5). 化合物结构经 ${ }^{1} \mathrm{H} \mathrm{NMR} 、{ }^{13} \mathrm{C} \mathrm{NMR} 、 \mathrm{IR}$ 和 HRMS 进行了表征, 并 对它们进行了抗血小板聚集活性测试，结果表明对抗血小板聚集显示一定的活性.
\end{abstract}

关键词＼cjkstart嘌呤衍生物; 合成; 结构表征; 抗血小板聚集活性

\section{Synthesis of 6-Alkylamino-2-ethylthiopurine Nucleoside Compounds and Their Antiplatelet Aggregation Activities}

\author{
Wang, Zijian ${ }^{a}$ \\ Li, Shunlai ${ }^{a}$ \\ Yu, Mingwu ${ }^{a}$ \\ Luo, Yuan ${ }^{b}$ \\ Wang, Yongan ${ }^{*, b}$ \\ $\mathrm{Du}$, Hongguang ${ }^{*, a}$ \\ ( ${ }^{a}$ Beijing University of Chemical Technology, Beijing 100029) \\ ( ${ }^{b}$ Institute of Pharmacology and Toxicology, Academy of Military Medical Sciences, State Key Laboratory of \\ Toxicology and Medical Countermeasures, Beijing 100850)
}

\begin{abstract}
Guanosine (1) as the starting material, reacted with acetic anhydride to obtain 2',3',5'-tri- $O$-acetyl-guanosine (2), which reacted with $\mathrm{TsCl}$ to obtain 2-amino-6-tosyl-9-(2',3',5'-tri- $O$-acetyl- $\beta$ - $D$-ribofuranosyl)purine (3). Compound 3 was diazotized with isoamyl nitrite and then reacted with dialkyl disulfides to afford 2-ethylthio-6-tosyl-9-(2',3',5'-tri- $O$-acetyl- $\beta$ - $D$ ribofuranosyl)purine (4). Finally, compounds 5 were acquired by aminolysis and deprotection of $\mathbf{4}$. Ten new compounds were synthesized and all compounds were characterized with ${ }^{1} \mathrm{H}$ NMR, ${ }^{13} \mathrm{C}$ NMR, IR and HRMS. Meanwhile, their antiplatelet aggregation rate was measured. The results show that these compounds have a certain antiplatelet aggregation activity.
\end{abstract}

Keywords purine derivatives; synthesis; structure characterization; antiplatelet aggregation activity

嘌呤衍生物不仅在生命体遗传、代谢等生理活动中 发挥着重要作用, 而且还具有抗癌、抗肿瘤和抗血栓等 重要活性 ${ }^{[1,2]}$. 对于由血小板聚集引起的疾病, 现在临床 用的药物如阿司匹林、氯吡格雷等都有一定的副作用. 由于嘌呤类化合物具有特殊的生理功能和药理作用，引 起了人们越来越多的关注 ${ }^{[3,4]}$. 因此, 对嘌呤衍生物的合 成及其抗血小板聚集作用的研究具有重要的意义.

2006 年, Katrin 等 ${ }^{[5]}$ 以 2-氨基-6-氯鸟嘌呤为原料,
对其 $2 、 6$ 和 9 位进行了结构修饰, 合成了一系列嘌呤化 合物，同时测定了它们的抗血小板聚集活性. 结果表明, 当嘌呤核苷的 6 位为烷氨基时抗血小板聚集活性最好.

近几年, 本课题组以嘌呤及嘌呤核苷为原料, 通过 在 2-位引入烷氧基或烷硫基，6-位引入烷氨基，合成了 一系列化合物, 对它们的抗血小板聚集活性进行了研 究 ${ }^{[6-9]}$, 结果发现部分化合物显示较好的抗血小板聚集 活性，如 BF061、BF0801 和 C4 等(图 1).

\footnotetext{
*E-mail:dhg@mail.buct.edu.cn; yonganw@126.com

Received April 23, 2015; revised June 4, 2015; published online June 12, 2015.

Project supported by the National Natural Science Foundation of China (No. 21272022).

国家自然科学基金(No. 21272022)资助项目.
} 


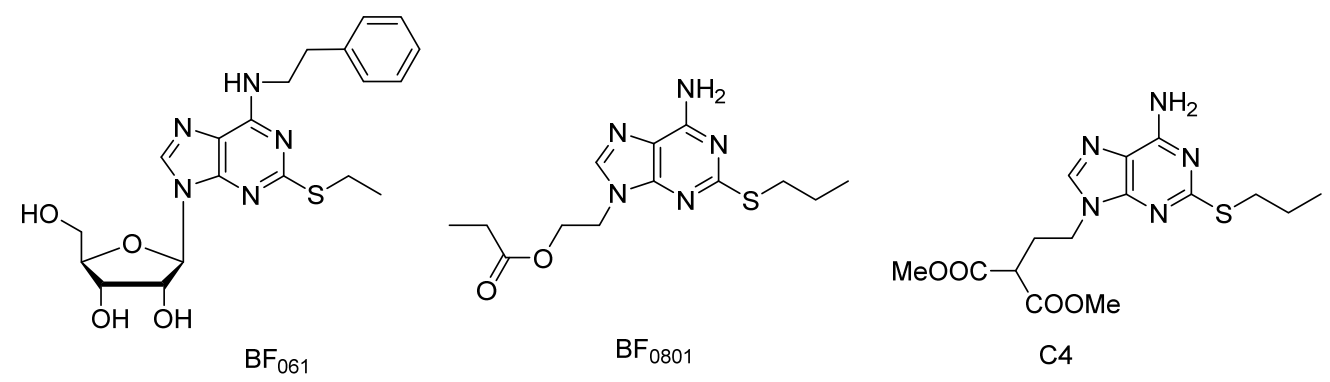

图 1 部分具有抗血小板聚集活性的嘌呤衍生物

Figure 1 Some purine derivatives with antiplatelet aggregation activity

本文以鸟苷(1)为原料, 经过糖环羟基保护得到 $2^{\prime}, 3^{\prime}, 5^{\prime}$-三- $O$-乙酰基鸟苷(2), 2 与对甲苯磺酰氯反应得到 9-(2',3',5'-三- $O$-乙酰基- $\beta$ - $D$-呋喃核糖)-2-氨基-6-对甲苯 磺酰氧基嘌呤 $(3), 3$ 经过 2-位氨基的重氮一乙硫化反应得 到了 9-(2', $3^{\prime}, 5^{\prime}$-三- $O$-乙酰基- $\beta$ - $D$-呋喃核糖)-6-对甲苯磺 酰氧基-2-乙硫基嘌呤(4). 4 经胺解和脱羟基保护反应得 到 6-烷氨基-2-乙硫基嘌呤核苷化合物(5)(合成路线见 Scheme 1), 其中, 嘌呤核苷 6 位取代基分别为 2 -氟苯乙 氨基、3-氟苯乙氨基、4-氟苯乙氨基、2-氯苯乙氨基、 3-氯苯乙氨基、4-氯苯乙氨基、哌啶基、吗啡啉基、4甲基哌嗪-1-基和噻吩乙氨基. 产物经过 ${ }^{1} \mathrm{H} N M R 、{ }^{13} \mathrm{C}$ NMR、IR 和 HRMS 进行表征, 并对它们进行了抗血小 板聚集活性的测试.

\section{1 结果与讨论}

\section{1 合成路线的选择}

鸟苷及其衍生物 6 位的差基进行氯化反应得到的化 合物是合成嘌呤衍生物重要的中间体, 常用的氯化试剂 是 $\mathrm{POCl}_{3}, \mathrm{SOCl}_{2}$, 三光气等. 本课题组 ${ }^{[10]}$ 对于中间体 2
的 6 位取代以往采用的方法是，在以四乙基氯化铵为相 转移催化剂、 $N, N$-二甲基苯胺为催化剂的条件下与 $\mathrm{POCl}_{3}$ 反应, 进行 6 位的氯化, 然后再与亲核试剂进行 取代反应, 实现 6 位的结构改造. 然而氯化反应收率较 低, 只有 $40 \%$. 原因可能是由于反应及后处理过程中强 烈的酸性条件使糖苷键容易断裂，导致副反应增多，且 $\mathrm{POCl}_{3}$ 具有一定毒性. 本研究小组由 2 开始, 经过 6 位羟 基氯化、2 位氨基重氮-乙硫化，与 2 -氟苯乙胺进行亲核 取代反应，最后进行脱羟基保护四步反应，得到 6-(2-氟 苯乙氨基)-2-乙硫基嘌呤核苷, 四步反应的总收率为 14\%. 本文以氢氧化钠为缚酸剂，4-二甲氨基吡啶 (DMAP) 为催化剂, 2 与对甲苯磺酰氯 $(\mathrm{TsCl})$ 常温反应制 备 3, 收率为 $79.9 \%$. 以此为中间体，经过 $\mathbf{4}$ 最后得到 6 位为烷氨基的嘌呤衍生物 $\mathbf{5}$. 以 2-氟苯乙胺为亲核试剂, 用该方法合成的 6-(2-氟苯乙氨基)-2-乙硫基嘌呤核苷的 四步反应的总收率为 $31 \%$.

\section{2 化合物 3 的合成工艺研究}

本文对化合物 3 的合成工艺进行了初步研究, 结果 列于表 1 . 由表 1 中 Entries $1 、 2$ 和 3 可以看出, 缚酸剂

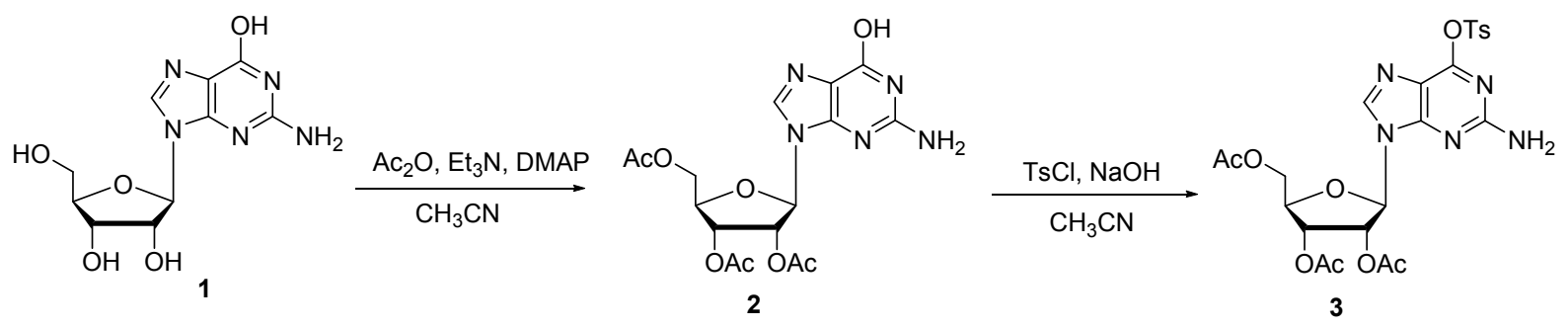<smiles>[R]Nc1nc(CC)nc2c1ncn2C1OC2(CO)C(CO)OC1C2O</smiles>

图式 1 6-烷氨基-2-乙硫基嘌呤核苷化合物的合成路线

Scheme 1 Synthetic route of 6-alkylamino-2-ethylthiopurine nucleoside compounds 
为 $\mathrm{Et}_{3} \mathrm{~N}$ 时, 只有以氯仿为溶剂反应才发生, 产率为 $55.2 \%$. 由表中 Entries 2、4、5 和 6 可以看出, 在四种缚 酸剂三乙胺、氢氧化钠、碳酸钠和碳酸氢钠中, 用氢氧 化钠反应收率最高, 为 $74.1 \%$. 由表中 Entries $4 、 7$ 和 8 可以看出, 溶剂为丙酮和乙腈的效果均较好. 从表中 Entries 8 和 9 可以看出, 催化剂 DMAP 的加入, 没有明 显改变反应的速率以及目标化合物的收率, 因此催化剂 DMAP 并不是该反应必须的条件, 无需加入. 本文还研 究了温度对反应的影响, 结果发现在加热回流的条件 下, 几乎不反应, 可能因为目标化合物热不稳定. 因此, 综合以上研究结果, 采用化合物 $2: \mathrm{TsCl}: \mathrm{NaOH}=1$ : $3: 3$ 的物料比投料, 溶剂为乙腈, 在室温反应 $10 \mathrm{~h}$ 为最 佳反应条件.

\section{3 结构表征分析}

论文对 10 个目标化合物进行了 ${ }^{1} \mathrm{H} \mathrm{NMR} 、{ }^{13} \mathrm{C}$ NMR、 IR 和 HRMS 的表征, 以化合物 5a 为例对其结构进行分 析. 在 $\mathbf{5 a}$ 的 ${ }^{1} \mathrm{H} \mathrm{NMR}$ 中(图 2) $\delta 8.22(\mathrm{~s}, 1 \mathrm{H}$ )为 8 位的 $\mathrm{H} ; \delta$ $8.07(\mathrm{~s}, 1 \mathrm{H})$ 为氨基的 $\mathrm{H} ; \delta 7.15(\mathrm{~m}, 2 \mathrm{H})$ 及 $\delta 7.25(\mathrm{~m}, 2 \mathrm{H})$ 为邻取代苯环上 $\mathrm{H}$ 的峰，由于受到氟的偶合影响导致这 两组峰均显示为多重峰; $\delta 5.43(\mathrm{~d}, 1 \mathrm{H}), 5.18(\mathrm{~d}, 1 \mathrm{H}), 5.08$ (brs, $1 \mathrm{H}$ )处为糖环的 3 个羟基中 $\mathrm{H}$ 的峰, $\delta 5.82(\mathrm{~d}, 1 \mathrm{H})$, $4.59(\mathrm{dd}, 1 \mathrm{H}), 4.14(\mathrm{dd}, 1 \mathrm{H}), 3.93(\mathrm{dd}, 1 \mathrm{H})$ 为糖环上 $1^{\prime} 、 2{ }^{\prime}$ 、 $3^{\prime}$ 和 4 '位 $\mathrm{H}$ 的峰; $\delta 3.50 \sim 3.70(\mathrm{~m}, 4 \mathrm{H})$ 是糖环上的 $5^{\prime}$ 位的 亚甲基和与 $\mathrm{NH}$ 相连的亚甲基中的 $\mathrm{H}$ 的峰. 然而, 糖环 上的亚甲基的两个 $\mathrm{H}$ 没有裂分为一个 $\mathrm{t}$ 峰，而是分别在 $\delta 3.55$ 和 3.65 处裂分为两个 ddd 峰. 分析原因是糖环上

表 1 化合物 3 合成反应条件的优化

Table 1 Optimization of the reaction conditions of synthesis compound 3

\begin{tabular}{clcccc}
\hline Entry & Solvent & Time $/ \mathrm{h}$ & $\mathrm{Base}$ & Ratio $(\mathbf{2}: \mathrm{TsCl}:$ Base : DMAP) & Yield/\% \\
\hline 1 & 氯仿 & 10 & $\mathrm{Et}_{3} \mathrm{~N}$ & $1: 3: 3: 0.13$ & 55.2 \\
2 & 丙酮 & 10 & $\mathrm{Et}_{3} \mathrm{~N}$ & $1: 3: 3: 0.13$ & - \\
3 & 乙腈 & 10 & $\mathrm{Et}_{3} \mathrm{~N}$ & $1: 3: 3: 0.13$ & - \\
4 & 丙酮 & 10 & $\mathrm{NaOH}$ & $1: 3: 3: 0.13$ & - \\
5 & 丙酮 & 10 & $\mathrm{Na}_{2} \mathrm{CO}_{3}$ & $1: 3: 3: 0.13$ & - \\
6 & 丙酮 & 10 & $\mathrm{NaHCO}_{3}$ & $1: 3: 3: 0.13$ & - \\
7 & 氯仿 & 10 & $\mathrm{NaOH}$ & $1: 3: 3: 0.13$ & 69.7 \\
8 & 乙腈 & 10 & $\mathrm{NaOH}$ & $1: 3: 3: 0.13$ & 79.9 \\
9 & 乙腈 & 10 & $\mathrm{NaOH}$ & $1: 3: 3: 0$ & \\
\hline
\end{tabular}

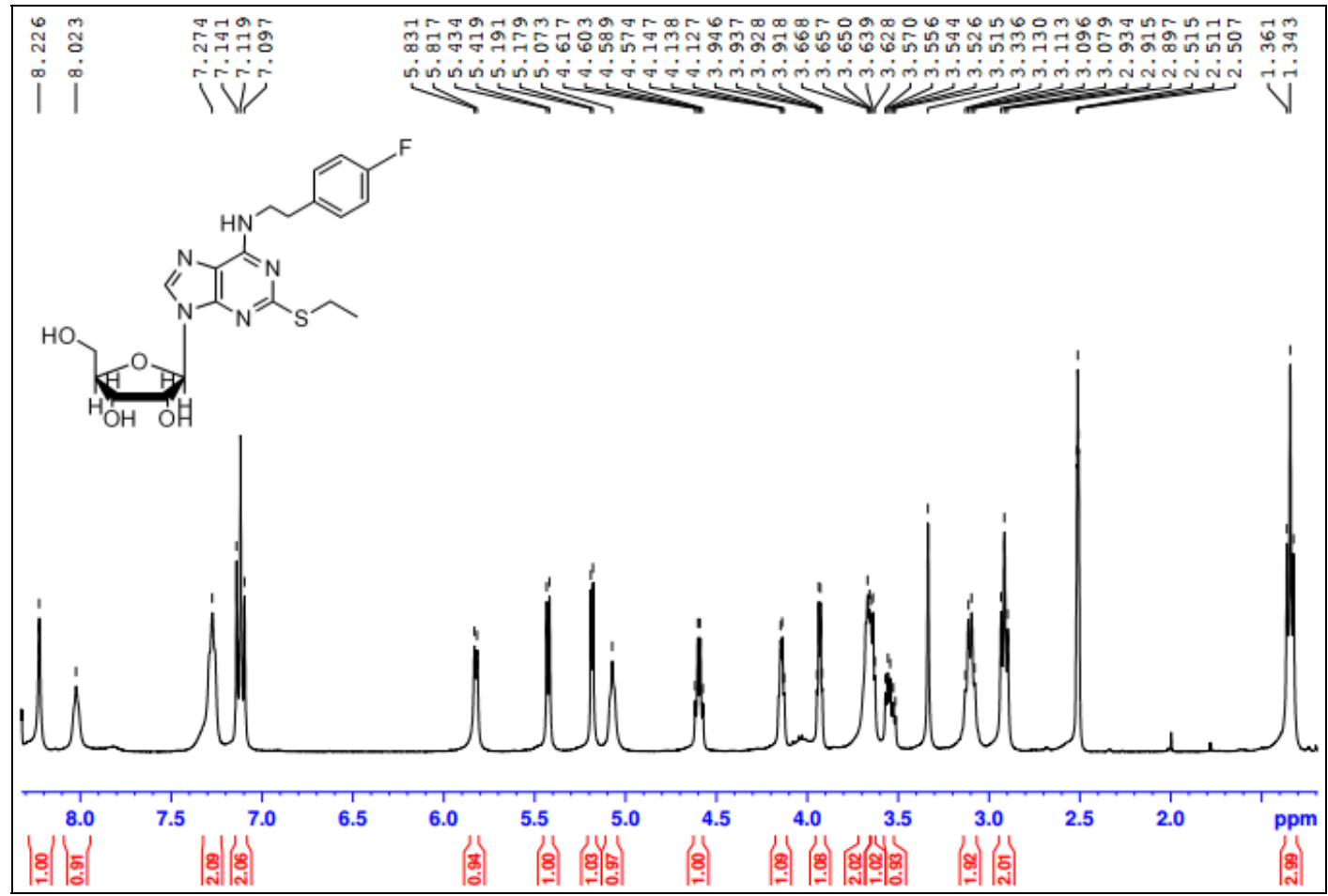

图 2 化合物 $5 \mathrm{a}$ 的核磁谱图

Figure $2{ }^{1} \mathrm{H}$ NMR of compound $\mathbf{5 a}$ 
手性碳原子的影响导致 $5^{\prime}$ 位的两个 $\mathrm{H}$ 发生偶合, 导致二 者的化学环境不同, 化学不等价, 裂分为 ddd 峰. $\delta 3.11$ (q, 2H)为乙硫基亚甲基上的 $\mathrm{H}$ 谱峰; $\delta 2.97(\mathrm{t}, 2 \mathrm{H})$ 处为与 苯环相连的亚甲基上 $\mathrm{H}$ 的峰; $\delta 1.34(\mathrm{t}, 3 \mathrm{H})$ 为甲基中 $\mathrm{H}$ 的峰.

在 ${ }^{13} \mathrm{C}$ NMR 中 $\delta 163.7,159.5,153.8,149.5,117.3$ 分 别为嘌呤环 $2 、 6 、 4 、 8$ 和 5 位的碳的吸收峰. 在 $\delta 162.0$ 处为苯环上与氟相连的碳的峰, 由于氟的强电负性使其 化学位移向低场移动. $\delta 131.2,128.2,126.0,124.2,115.1$ 处的 5 个 2 重峰为苯环上其余 5 个碳的峰, 原因是因为 氟原子会使与其相邻的碳发生裂分. $\delta 87.4,85.6,79.1$, $73.3,70.5$ 处的 5 个峰分别为糖环上 1'、2'、3'、4'和 $5^{\prime}$ 位的碳谱峰. $\delta 61.6$ 和 28.5 分别为苯乙氨基上与氨基和 苯环相连的亚甲基的碳谱峰; $\delta 24.7$ 和 15.0 分别为乙硫 基的亚甲基和甲基的碳谱峰.

在 IR (KBr)中, 在 $3332 \mathrm{~cm}^{-1}$ 处有一个较宽的吸收 峰, 这是嘌呤糖环上的 3 个羟基的 $\mathrm{O}-\mathrm{H}$ 伸缩振动峰和 仲胺的 $\mathrm{N}-\mathrm{H}$ 的伸缩振动峰发生重叠. 此外在 2928 $\mathrm{cm}^{-1}$ 处有一个较宽的吸收峰, 分析为甲基和亚甲基 $\mathrm{C}-$ $\mathrm{H}$ 的伸缩振动峰. 在 1611 和 $1491 \mathrm{~cm}^{-1}$ 为苯环的骨架振 动的特征吸收峰; 在 $1338 \mathrm{~cm}^{-1}$ 处为甲基和亚甲基的 $\mathrm{C}-\mathrm{H}$ 的面内弯曲振动峰; 在 1299 和 $1228 \mathrm{~cm}^{-1}$ 处为嘌 呤环的 $\mathrm{C}-\mathrm{N}$ 的伸缩振动峰; 在 $1109 \mathrm{~cm}^{-1}$ 处为苯环上 $\mathrm{C}-\mathrm{F}$ 的振动峰; 在 $982 \mathrm{~cm}^{-1}$ 处很强的吸收峰为嘌呤五 元单氧环 $\mathrm{C}-\mathrm{O}-\mathrm{C}$ 的伸缩振动峰; 在 $639 \mathrm{~cm}^{-1}$ 处为乙 硫基中 $\mathrm{S}-\mathrm{C}$ 的振动峰.

同时, HRMS (ESI) calcd for $\mathrm{C}_{19} \mathrm{H}_{22} \mathrm{FN}_{5} \mathrm{O}_{4} \mathrm{~S}[\mathrm{M}+\mathrm{H}]^{+}$ 435.1377, found 435.1376 表明了 5a 的结构.

\section{4 抗血小板聚集活性的测试}

本文对 10 个 6-烷氨基-2-乙硫基嘌呤核苷化合物进
行了抗血小板聚集活性，结果列于表 2. 大鼠腹腔注射 $10 \%$ 乌拉坦麻醉, 心脏取血, 用 $3.8 \%$ 柠檬酸钠抗凝(1： 9), 室温下 $2000 \mathrm{r} / \mathrm{min}$, 离心 $10 \mathrm{~min}$. 小心吸取上层血浆 即为富血小板血浆(有溶血现象弃去不用); 剩余样本以 $3500 \mathrm{r} / \mathrm{min}$ 离心 $10 \mathrm{~min}$, 再吸取上层血浆即为贫血小板 血浆. 聚集仪预热至 $37{ }^{\circ} \mathrm{C}$, 用 PPP 调整透光率为 $100 \%$. 取 PRP $490 \mu \mathrm{L}$ 置于比浊管中, 实验组加入 $5 \mu \mathrm{L}$ 待试药 品, 对照组加入等容积对照品, 温育 $5 \mathrm{~min}$ 后于 PRP 管 中加入诱导剂 $\mathrm{ADP} / \mathrm{AA} / \mathrm{COLL}$ 诱导血小板聚集. 记录 5 min 内图形变化, 读取最大聚集率. 以血小板最大聚集 率为观测指标, 并计算血小板聚集抑制率: 抑制率 $(\%)=[($ 空白对照组血小板最大聚集率一实验组血小板 最大聚集率)/空白对照组血小板最大聚集率] $\times 100 \%$. 在二磷酸腺苷(adenosine diphosphate, ADP)、花生四烯酸 及胶原诱导血小板聚集模型上，依据药物作用机理，分 别采用替卡格雷、阿司匹林及替罗非班作为阳性对照药. 在给药浓度恒为 $30 \mu \mathrm{mol} / \mathrm{L}$. 测试结果表明: (1)当 6 位为 氟或氯取代苯乙胺基时, 对位苯乙胺取代的嘌呤核苷的 抗血小板抑制作用要强于邻位和间位苯乙胺取代的嘌 呤核苷的抗血小板抑制作用; (2) 6 位为苯乙胺的衍生物 取代基时，嘌呤核苷对于 ADP 诱导的血小板的抑制作 用最好; 6 位为杂环取代时，嘌呤核草对于花生四烯酸 诱导的血小板的抑制作用最好; 所有化合物对于胶原诱 导的血小板抑制作用均不明显.

\section{2 结论}

以鸟苷为原料，经过糖环羟基保护、6-位羟基对甲 苯磺酰化、2-位氨基重氮-乙硫化、6-位胺解以及糖环的 脱保护等 5 步反应合成了 10 个 6 -烷氨基-2-乙硫基嘌呤

表 2 化合物 $\mathbf{5}$ 的抗血小板聚集活性数据

Table 2 The antiplatelet aggregation activity data of compounds 5

\begin{tabular}{|c|c|c|c|c|c|c|}
\hline \multirow{2}{*}{ Compd. } & \multicolumn{2}{|c|}{ ADP } & \multicolumn{2}{|c|}{ Arachidonic acid } & \multicolumn{2}{|c|}{ Collagen } \\
\hline & $\mathrm{PAR}^{a} / \%$ & $\mathrm{PAIR}^{b} / \%$ & $\operatorname{PAR}^{a} / \%$ & $\mathrm{PAIR}^{b} / \%$ & $\mathrm{PAR}^{a} / \%$ & $\operatorname{PAIR}^{b} / \%$ \\
\hline $5 a$ & 51 & 21.5 & 49 & - & 48 & 22.6 \\
\hline $5 b$ & 49 & 24.6 & 46 & - & 40 & 35.5 \\
\hline $5 c$ & 12 & 52.0 & 20 & 42.9 & 39 & 37.1 \\
\hline $5 d$ & 50 & 23.1 & 30 & 25 & 61 & 1.0 \\
\hline $5 e$ & 50 & 23.1 & 49 & - & 69 & - \\
\hline $5 f$ & 10 & 60.0 & 25 & 28.6 & 50 & 19.4 \\
\hline $5 g$ & 45 & - & 30 & 43.4 & 38 & 50.0 \\
\hline $5 \mathrm{~h}$ & 60 & - & 25 & 52.8 & 50 & 27.5 \\
\hline $5 i$ & 41 & 8.9 & 20 & 62.3 & 38 & 38.7 \\
\hline $5 \mathbf{j}$ & 26 & 42.2 & 40 & 24.5 & 47 & 24.2 \\
\hline 替卡格雷 & 0 & 100 & - & - & - & - \\
\hline 阿司匹林 & - & - & 14 & 73.6 & - & - \\
\hline 替罗非班 & - & - & - & - & 0 & 100 \\
\hline
\end{tabular}

聚集率; ${ }^{b}$ 抑制率. 
核苷化合物，进行了抗血小板聚集活性测试. 结果表明, 化合物 5 对于 ADP、花生四烯酸和胶原等三种诱导剂诱 导的血小板聚集均有一定的抑制作用, 但活性都不显 著.

\section{3 实验部分}

\section{1 仪器与试剂}

鸟苷, 河南新乡拓新化工厂; 硅胶( $200 \sim 300$ 目), 青岛海洋化工厂; 丙酩、甲醇、乙腈及亚硝酸异戊酯等 为化学纯, 北京化学试剂; 二乙基二硫醚, 滕州吉田香 料股份有限公司; 化合物 2 按文献[10]方法制备; XT-4A 型显微熔点测定仪, 上海精密科学仪器有限公司(温度 计未校正); Bruker $400 \mathrm{MHz}$ 核磁共振仪, 德国 Bruker 公 司; Agilent QTOF-6530 型高分辨质谱仪, 美国 Agilent 公司.

\section{2 实验方法}

3.2.19-( 2 ', 3', $5^{\prime}$-三- $O$-乙酰基- $\beta$ - $D$-呋喃核糖)-2-氨基6-对甲苯磺酰氧基嘌呤的合成

将无水乙腈 $(20 \mathrm{~mL})$ 加入圆底烧瓶中, 室温搅拌下, 依次加入 $2(1.0 \mathrm{~g}, 2.4 \mathrm{mmol}) 、 \mathrm{TsCl}(1.40 \mathrm{~g}, 7.3 \mathrm{mmol})$ 和 $\mathrm{NaOH}$ (0.29 g, $7.3 \mathrm{mmol})$, 反应 $10 \mathrm{~h}$, 减压旋除乙腈, 用 乙酸乙酯复溶, 加入 $10 \mathrm{~mL}$ 水震荡后分液, 水相用乙酸 乙酯萃取, 合并有机相. 用无水 $\mathrm{MgSO}_{4}$ 干燥, 经过滤, 减压蒸掉溶剂后, 剩余物经柱色谱分离(硅胶, EtOAc-P.E., 1：1)得到 $1.10 \mathrm{~g}$ 白色晶体 3, 收率 79.9\%. m.p. $74 \sim 76{ }^{\circ} \mathrm{C} ;{ }^{1} \mathrm{H}$ NMR $\left(\mathrm{CDCl}_{3}, 400 \mathrm{MHz}\right) \delta: 8.02$ (d, $J=8.3 \mathrm{~Hz}, 2 \mathrm{H}, \mathrm{ArH}), 7.80$ (s, 1H, H-8), 7.36 (d, $J=8.2$ $\mathrm{Hz}, 2 \mathrm{H}, \mathrm{ArH}), 5.99$ (d, $\left.J=4.9 \mathrm{~Hz}, 1 \mathrm{H}, \mathrm{H}-1^{\prime}\right), 5.93$ (dd, $J=$ 5.0, $\left.5.2 \mathrm{~Hz}, 1 \mathrm{H}, \mathrm{H}-2^{\prime}\right), 5.75$ (dd, $\left.J=4.9,5.0 \mathrm{~Hz}, 1 \mathrm{H}, \mathrm{H}-3^{\prime}\right)$, 5.16 (s, 2H, NH 2$), 4.44$ (m, 1H, H-4'), 4.40 (m, 1H, H-5'a), 4.35 (m, 1H, H-5'b), 2.45 (s, 3H, $\left.\mathrm{ArCH}_{3}\right), 2.13$ (s, 3H, $\left.\mathrm{COCH}_{3}-2^{\prime}\right), 2.09$ (s, 3H, $\left.\mathrm{COCH}_{3}-3^{\prime}\right), 2.06$ (s, 3H,

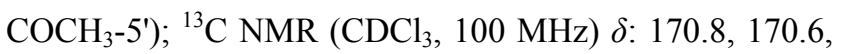
$169.8,158.9,155.3,154.6,141.3,140.7,133.6,130.0$, $129.7,129.0,128.7,116.6,86.6,80.0,72.8,70.5,63.0$, 21.7, 21.3, 20.7, 20.5.

$3.2 .29-\left(2^{\prime}, 3^{\prime}, 5^{\prime}-\right.$ 三- $O$-乙酰基- $\beta$ - $D$-呋喃核糖)-6-对甲 苯磺酰氧基-2-乙硫基嘌呤的合成

将 3 (2.4 g, $4.26 \mathrm{mmol})$ 和二乙基二硫醚(12.78 $\mathrm{mmol}$ )加入到 $30 \mathrm{~mL}$ 无水乙腈中, 然后加入亚硝酸异戊 酯(1.50 g, $12.78 \mathrm{mmol})$, 在 $65{ }^{\circ} \mathrm{C}$ 水中超声 $1 \mathrm{~h}$. 减压蒸 掉溶剂和未反应的原料, 剩余物经柱色谱分离(硅胶, EtOAc-P.E., 2：3 1：1)后得到 $1.22 \mathrm{~g}$ 淡黄色油状液体 4, 收率 47\%; ${ }^{1} \mathrm{H} \mathrm{NMR}\left(\mathrm{CDCl}_{3}, 400 \mathrm{MHz}\right) \delta: 8.03$ (d, $J=$ $8.0 \mathrm{~Hz}, 2 \mathrm{H}, \mathrm{ArH}), 8.02$ (s, 1H, H-8), 7.38 (d, $J=8.4 \mathrm{~Hz}$,
2H, ArH), 6.12 (d, $\left.J=5.2 \mathrm{~Hz}, 1 \mathrm{H}, \mathrm{H}-1^{\prime}\right), 5.59$ (dd, $J=5.2$, $\left.5.2 \mathrm{~Hz}, 1 \mathrm{H}, \mathrm{H}-2^{\prime}\right), 5.85$ (dd, $\left.J=5.2,5.2 \mathrm{~Hz}, 1 \mathrm{H}, \mathrm{H}-3^{\prime}\right), 4.43$ (m, 1H, H-4'), 4.41 (m, 1H, H-5'a), 4.32 (ddd, $J=4.0,4.6$, $12.0 \mathrm{~Hz}, 1 \mathrm{H}, \mathrm{H}-5$ 'b), 3.09 (t, J=7.1 Hz, 2H, $\mathrm{SCH}_{2}$ ), 2.47 $\left(\mathrm{s}, 3 \mathrm{H}, \mathrm{ArCH}_{3}\right), 2.14\left(\mathrm{~s}, 3 \mathrm{H}, \mathrm{COCH}_{3}-2\right.$ ), $2.10(\mathrm{~s}, 3 \mathrm{H}$, $\left.\mathrm{COCH}_{3}-3^{\prime}\right), 2.09$ (s, 3H, $\left.\mathrm{COCH}_{3}-5^{\prime}\right), 1.05$ (t, $J=7.3 \mathrm{~Hz}$, $\left.3 \mathrm{H}, \mathrm{CH}_{3}\right) ;{ }^{13} \mathrm{C} \mathrm{NMR}\left(\mathrm{CDCl}_{3}, 100 \mathrm{MHz}\right) \delta: 170.8,170.2$, $169.0,158.2,155.5,154.0,140.6,140.2,133.3,130.0$, $129.8,129.2,128.2,116.5,85.5,81.2,72.8,70.0,62.1$, 28.5, 21.7, 21.5, 20.7, 20.1, 14.3.

\section{2 .3 化合物 $\mathbf{5}$ 的合成}

将 4 (1.22 g, $2 \mathrm{mmol})$ 溶于 $20 \mathrm{~mL}$ 无水乙醇中, 依次 加入三乙胺 $(0.40 \mathrm{~g}, 4 \mathrm{mmol})$ 和相应的胺 $(4 \mathrm{mmol})$, 回流 反应 $1 \mathrm{~h}, \mathrm{TLC}(\mathrm{MeOH}-\mathrm{EtOAc}, V: V=1: 15)$ 监测, 原料 消失后停止加热. 待反应液冷却后加入催化量的甲醇钠 搅拌, TLC 监测反应完全后加入适量水, 减压蒸掉溶剂, 剩余物用乙酸乙酯萃取 3 次, 合并有机相. 有机相用稀 盐酸、 $5 \% \mathrm{NaHCO}_{3}$ 、饱和食盐水和水洗涤后, 用无水 $\mathrm{MgSO}_{4}$ 干燥. 过滤, 减压蒸掉溶剂. 剩余物经柱色谱分 离(MeOH-EtOAc, $V: V=1: 30)$ 得到白色晶体 5 .

6-(2-氟苯乙氨基)-2-乙硫基嘌呤核苷(5a): 白色固 体, 收率 80\%. m.p. 90 92 ${ }^{\circ} \mathrm{C} ;{ }^{1} \mathrm{H}$ NMR (DMSO- $d_{6}, 400$ $\mathrm{MHz}) \delta: 8.22$ (s, 1H, H-8), 8.07 (br s, 1H, NH), 7.20 (m, 4H, ArH), 5.82 (d, $\left.J=5.9 \mathrm{~Hz}, 1 \mathrm{H}, \mathrm{H}-1^{\prime}\right), 5.43$ (d, $J=6.2$ $\mathrm{Hz}, 1 \mathrm{H}, \mathrm{OH}), 5.18$ (d, $J=4.8 \mathrm{~Hz}, 1 \mathrm{H}, \mathrm{OH}), 5.08$ (br s, $1 \mathrm{H}$, $\mathrm{OH}), 4.59$ (dd, $\left.J=6.0,11.6 \mathrm{~Hz}, 1 \mathrm{H}, \mathrm{H}-2^{\prime}\right), 4.14$ (dd, $J=$ 4.0, 7.6 Hz, 1H, H-3'), 3.93 (dd, $J=3.6,7.2 \mathrm{~Hz}, 1 \mathrm{H}, \mathrm{H}-4$ '), $3.70 \sim 3.79\left(\mathrm{~m}, 2 \mathrm{H}, \mathrm{NCH}_{2}\right), 3.64 \sim 3.67(\mathrm{~m}, 1 \mathrm{H}, \mathrm{H}-5 \mathrm{a})$, $3.54 \sim 3.56$ (m, 1H, H-5'b), 3.11 (q, J=7.0 Hz, 2H, $\mathrm{SCH}_{2}$ ), $2.97\left(\mathrm{t}, J=7.2 \mathrm{~Hz}, 2 \mathrm{H}, \mathrm{NCH}_{2} \mathrm{CH}_{2}\right), 1.34$ (t, $J=7.2 \mathrm{~Hz}, 3 \mathrm{H}$, $\left.\mathrm{CH}_{3}\right) ;{ }^{13} \mathrm{C}$ NMR (DMSO- $\left.d_{6}, 100 \mathrm{MHz}\right) \delta: 163.7,162.0$, $159.5,153.8,149.5,138.6,131.2,131.1,128.3,128.2$, $126.1,125.9,124.3,124.2,117.3,115.2,115.0,87.4,85.6$, 79.1, 73.3, 70.5, 61.6, 28.5, 24.7, 15.0; IR (KBr) v: 3332, 2928, 1611, 1491, 1339, 1299, 1110, $1078 \mathrm{~cm}^{-1}$; HRMS (ESI) calcd for $\mathrm{C}_{20} \mathrm{H}_{25} \mathrm{FN}_{5} \mathrm{O}_{4} \mathrm{~S}[\mathrm{M}+\mathrm{H}]^{+} 450.1612$, found 450.1606 .

6-(3-氟苯乙氨基)-2-乙硫基嘌呤核苷(5b): 白色固 体, 收率 $82 \%$. m.p. $124 \sim 126{ }^{\circ} \mathrm{C} ;{ }^{1} \mathrm{H}$ NMR (DMSO- $d_{6}$, $400 \mathrm{MHz}) \delta: 8.23$ (s, 1H, H-8), 8.04 (br s, 1H, NH), 7.2 (m, 4H, ArH), 5.82 (d, J=5.6 Hz, 1H, H-1'), 5.43 (d, $J=$ $6.0 \mathrm{~Hz}, 1 \mathrm{H}, \mathrm{OH}), 5.18$ (d, J=4.6 Hz, 1H, OH), 5.07 (br s, $1 \mathrm{H}, \mathrm{OH}), 4.59$ (dd, $\left.J=5.6,10.8 \mathrm{~Hz}, 1 \mathrm{H}, \mathrm{H}-2^{\prime}\right), 4.14$ (d, $J=$ $\left.3.4 \mathrm{~Hz}, 1 \mathrm{H}, \mathrm{H}-3^{\prime}\right), 3.93$ (d, J=3.5 Hz, 1H, H-4'), 3.69 $3.83\left(\mathrm{~m}, 2 \mathrm{H}, \mathrm{NCH}_{2}\right), 3.63 \sim 3.66(\mathrm{~m}, 1 \mathrm{H}, \mathrm{H}-5 \mathrm{\prime}), 3.54 \sim$ 
3.60 (m, 1H, H-5'b), 3.11 (q, $J=6.7 \mathrm{~Hz}, 2 \mathrm{H}, \mathrm{SCH}_{2}$ ), 2.95 (t, $\left.J=7.2 \mathrm{~Hz}, 2 \mathrm{H}, \mathrm{NCH}_{2} \mathrm{CH}_{2}\right), 1.34$ (t, $J=7.2 \mathrm{~Hz}, 3 \mathrm{H}$, $\mathrm{CH}_{3}$ ); ${ }^{13} \mathrm{C}$ NMR (DMSO- $\left.d_{6}, 100 \mathrm{MHz}\right) \delta: 163.7,163.4$, $161.0,153.8,149.5,142.5,138.6,130.2,130.1,124.8$, $117.3,115.4,115.2,112.9,112.7,87.4,85.5,73.3,70.5$, 61.6, 59.7, 41.0, 34.6, 24.7, 20.7, 15.0, 14.0; IR (KBr) $v$ : 3102, 2963, 2292, 2857, 1578, 1194, 1343, 1256, 1116 $\mathrm{cm}^{-1}$; HRMS (ESI) calcd for $\mathrm{C}_{20} \mathrm{H}_{25} \mathrm{FN}_{5} \mathrm{O}_{4} \mathrm{~S}[\mathrm{M}+\mathrm{H}]^{+}$ 450.1612, found 450.1603 .

6-(4-氟苯乙氨基)-2-乙硫基嘌呤核苷 $(\mathbf{5 c})$ : 白色固 体, 收率 61\%. m.p. 141 142 ${ }^{\circ} \mathrm{C} ;{ }^{1} \mathrm{H}$ NMR (DMSO-d $d_{6}$, $400 \mathrm{MHz}) \delta: 8.23$ (s, 1H, H-8), 8.03 (br s, $1 \mathrm{H}, \mathrm{NH}), 7.31$ (m, 4H, ArH), 5.83 (d, J=5.7 Hz, 1H, H-1'), 5.42 (d, $J=$ $5.1 \mathrm{~Hz}, 1 \mathrm{H}, \mathrm{OH}), 5.18$ (br s, $1 \mathrm{H}, \mathrm{OH}), 5.07$ (br s, $1 \mathrm{H}, \mathrm{OH}$ ), 4.59 (d, $\left.J=4.8 \mathrm{~Hz}, 1 \mathrm{H}, \mathrm{H}-2^{\prime}\right), 4.14 \sim 4.33$ (m, 1H, H-3'), 3.93 (dd, $\left.J=3.6,7.2 \mathrm{~Hz}, 1 \mathrm{H}, \mathrm{H}-4^{\prime}\right), 3.67 \sim 3.80(\mathrm{~m}, 2 \mathrm{H}$, $\left.\mathrm{NCH}_{2}\right), 3.62 \sim 3.65(\mathrm{~m}, 1 \mathrm{H}, \mathrm{H}-5$ 'a), $3.54 \sim 3.59(\mathrm{~m}, 1 \mathrm{H}$, H-5'b), 3.10 (q, $J=6.8 \mathrm{~Hz}, 2 \mathrm{H}, \mathrm{SCH}_{2}$ ), 2.92 (t, $J=7.3 \mathrm{~Hz}$, $2 \mathrm{H}, \mathrm{NCH}_{2} \mathrm{CH}_{2}$ ), 1.34 (t, $\left.J=7.2 \mathrm{~Hz}, 3 \mathrm{H}, \mathrm{CH}_{3}\right) ;{ }^{13} \mathrm{C} \mathrm{NMR}$ (DMSO- $\left.d_{6}, 100 \mathrm{MHz}\right) \delta: 163.7,153.8,149.4,138.6,138.5$, $130.7,130.5,128.2,117.3,87.4,85.5,73.3,70.5,61.6$, 41.1, 34.2, 25.0, 24.7, 15.0; IR (KBr) v: 3353, 2927, 1619, 1582, 1510, 1477, 1341, 1296, 1225, $1081 \mathrm{~cm}^{-1}$; HRMS (ESI) calcd for $\mathrm{C}_{20} \mathrm{H}_{25} \mathrm{FN}_{5} \mathrm{O}_{4} \mathrm{~S}[\mathrm{M}+\mathrm{H}]^{+}$450.1612, found 450.1605 .

6-(2-氯苯乙氨基)-2-乙硫基嘌呤核苷 $(\mathbf{5 d})$ : 白色固 体, 收率 79\%. m.p. 93 95 ${ }^{\circ} \mathrm{C} ;{ }^{1} \mathrm{H}$ NMR (DMSO- $d_{6}, 400$ MHz) $\delta: 8.23$ (s, 1H, H-8), 8.08 (br s, 1H, NH), 7.44 7.23 (m, 4H, ArH), 5.82 (d, $\left.J=5.6 \mathrm{~Hz}, 1 \mathrm{H}, \mathrm{H}-1^{\prime}\right), 5.43(\mathrm{~d}, J=$ $4.8 \mathrm{~Hz}, 1 \mathrm{H}, \mathrm{OH}), 5.19$ (br s, 1H, OH), 5.08 (br s, 1H, OH), $4.59 \sim 4.71$ (m, 1H, H-2'), 4.14 (br s, 1H, H-3'), 3.93 4.07 (m, 1H, H-4'), 3.73 $3.81\left(\mathrm{~m}, 2 \mathrm{H}, \mathrm{NCH}_{2}\right), 3.65 \sim 3.70(\mathrm{~m}$, $\left.1 \mathrm{H}, \mathrm{H}-5^{\prime} \mathrm{a}\right), 3.54 \sim 3.59$ (m, 1H, H-5'b), 3.10 (q, $J=8.1 \mathrm{~Hz}$, $2 \mathrm{H}, \mathrm{SCH}_{2}$ ), $3.06\left(\mathrm{t}, J=7.1 \mathrm{~Hz}, 2 \mathrm{H}, \mathrm{NCH}_{2} \mathrm{CH}_{2}\right), 1.33$ (t, $\left.J=7.3 \mathrm{~Hz}, 3 \mathrm{H}, \mathrm{CH}_{3}\right) ;{ }^{13} \mathrm{C}$ NMR (DMSO- $\left.d_{6}, 100 \mathrm{MHz}\right) \delta$ : $163.7,153.8,149.5,138.6,136.9,133.2,131.0,129.2$, $128.1,127.2,117.3,87.4,85.5,79.1,73.3,70.5,61.6,32.8$, 24.8, 15.0; IR (KBr) v: 3362, 2926, 1619, 1583, 1476, 1337, $1299 \mathrm{~cm}^{-1}$; HRMS (ESI) calcd for $\mathrm{C}_{20} \mathrm{H}_{25} \mathrm{ClN}_{5} \mathrm{O}_{4} \mathrm{~S}$ $[\mathrm{M}+\mathrm{H}]^{+}$466.1317, found 466.1310.

6-(3-氯苯乙氨基)-2-乙硫基嘌呤核苷(5e): 白色固 体, 收率 83\%. m.p. 92 94 ${ }^{\circ} \mathrm{C} ;{ }^{1} \mathrm{H}$ NMR (DMSO- $d_{6}, 400$ MHz) $\delta: 8.23$ (s, 1H, H-8), 8.05 (br s, 1H, NH), 7.28 (m, 4H, ArH), 5.82 (d, $J=5.6 \mathrm{~Hz}, 1 \mathrm{H}, \mathrm{H}-1$ '), 5.43 (d, $J=6.1$ $\mathrm{Hz}, 1 \mathrm{H}, \mathrm{OH}), 5.18$ (d, J=4.6 Hz, 1H, OH), 5.07 (br s, $1 \mathrm{H}$,
OH), 4.59 (dd, $\left.J=5.6,11.2 \mathrm{~Hz}, 1 \mathrm{H}, \mathrm{H}-2^{\prime}\right), 4.14$ (d, $J=3.4$ Hz, 1H, H-3'), 3.93 (d, J=3.5 Hz, 1H, H-4'), $3.69 \sim 3.82$ (m, $2 \mathrm{H}, \mathrm{NCH}_{2}$ ), $3.65 \sim 3.68(\mathrm{~m}, 1 \mathrm{H}, \mathrm{H}-5$ 'a), 3.54 3.61 (m, 1H, H-5'b), 3.11 (q, J=6.8 Hz, 2H, $\mathrm{SCH}_{2}$ ), 2.94 (t, $\left.J=7.0 \mathrm{~Hz}, 2 \mathrm{H}, \mathrm{NCH}_{2} \mathrm{CH}_{2}\right), 1.35\left(\mathrm{t}, J=7.1 \mathrm{~Hz}, 3 \mathrm{H}, \mathrm{CH}_{3}\right)$; ${ }^{13} \mathrm{C}$ NMR (DMSO- $\left.d_{6}, 100 \mathrm{MHz}\right) \delta: 163.7,153.8,149.5$, $142.1,138.6,132.9,130.1,128.5,127.4,126.0,117.3$, $87.4,85.5,73.3,70.5,61.6,41.0,34.5,24.7,15.0$; IR (KBr) $v$ : 3332, 2927, 2868, 1617, 1476, 1428, 1338, 1299 , 1228, $1079 \mathrm{~cm}^{-1}$; HRMS (ESI) calcd for HRMS (ESI) calcd for $\mathrm{C}_{20} \mathrm{H}_{25} \mathrm{ClN}_{5} \mathrm{O}_{4} \mathrm{~S}[\mathrm{M}+\mathrm{H}]^{+}$466.1317, found 466.1306 .

6-(4-氯苯乙氨基)-2-乙硫基嘌呤核苷(5f)：白色固 体, 收率 67\%. m.p. $161 \sim 163{ }^{\circ} \mathrm{C} ;{ }^{1} \mathrm{H}$ NMR (DMSO- $d_{6}$, $400 \mathrm{MHz}) \delta: 8.23$ (s, 1H, H-8), 8.03 (br s, 1H, NH), 7.31 7.56 (m, 4H, ArH), 5.83 (d, $\left.J=5.7 \mathrm{~Hz}, 1 \mathrm{H}, \mathrm{H}-1^{\prime}\right), 5.42(\mathrm{~d}$, $J=5.1 \mathrm{~Hz}, 1 \mathrm{H}, \mathrm{OH}), 5.18$ (br s, 1H, OH), 5.07 (br s, $1 \mathrm{H}$, $\mathrm{OH}), 4.59\left(\mathrm{~d}, J=4.8 \mathrm{~Hz}, 1 \mathrm{H}, \mathrm{H}-2^{\prime}\right), 4.14 \sim 4.31(\mathrm{~m}, 1 \mathrm{H}$, H-3'), 3.93 (dd, $\left.J=3.6,7.2 \mathrm{~Hz}, 1 \mathrm{H}, \mathrm{H}-4^{\prime}\right), 3.67 \sim 3.84$ (m, $\left.2 \mathrm{H}, \mathrm{NCH}_{2}\right), 3.64 \sim 3.66(\mathrm{~m}, 1 \mathrm{H}, \mathrm{H}-5 \mathrm{\prime} \mathrm{a}), 3.54 \sim 3.59(\mathrm{~m}$, $1 \mathrm{H}, \mathrm{H}-5 \mathrm{'}$ ) $), 3.10$ (q, $J=6.8 \mathrm{~Hz}, 2 \mathrm{H}, \mathrm{SCH}_{2}$ ), 2.92 (t, $J=7.3$ $\left.\mathrm{Hz}, 2 \mathrm{H}, \mathrm{NCH}_{2} \mathrm{CH}_{2}\right), 1.34\left(\mathrm{t}, J=7.2 \mathrm{~Hz}, 3 \mathrm{H}, \mathrm{CH}_{3}\right) ;{ }^{13} \mathrm{C}$ NMR (DMSO- $\left.d_{6}, 100 \mathrm{MHz}\right) \delta$ : 163.7, 153.8, 149.4, 138.6, $138.5,130.7,130.5,128.2,117.3,87.4,85.5,73.3,70.5$, 61.6, 41.1, 34.2, 25.0, 24.7, 15.0; IR (KBr) v: 3348, 2926, 1618, 1582, 1476, 1339, $1297 \mathrm{~cm}^{-1}$; HRMS (ESI) calcd for $\mathrm{C}_{20} \mathrm{H}_{24} \mathrm{ClN}_{5} \mathrm{O}_{4} \mathrm{~S}[\mathrm{M}+\mathrm{H}]^{+}$466.1317, found 466.1307.

6-哌啶基-2-乙硫基嘌呤核苷 $(\mathbf{5 g})$ : 白色固体, 收率 69\%. m.p. 79 81 ${ }^{\circ} \mathrm{C} ;{ }^{1} \mathrm{H}$ NMR (DMSO- $\left.d_{6}, 400 \mathrm{MHz}\right) \delta$ : 8.25 (s, $1 \mathrm{H}, \mathrm{H}-8), 5.85$ (d, $\left.J=5.8 \mathrm{~Hz}, 1 \mathrm{H}, \mathrm{H}-\mathrm{l}^{\prime}\right), 5.43$ (d, $J=6.1 \mathrm{~Hz}, 1 \mathrm{H}, \mathrm{OH}), 5.18(\mathrm{~d}, J=4.9 \mathrm{~Hz}, 1 \mathrm{H}, \mathrm{OH}), 5.06(\mathrm{t}$, $J=5.4 \mathrm{~Hz}, 1 \mathrm{H}, \mathrm{OH}), 4.56$ (dd, $\left.J=6.0,11.2 \mathrm{~Hz}, 1 \mathrm{H}, \mathrm{H}-2^{\prime}\right)$, $4.20 \sim 4.48\left(\mathrm{~m}, 4 \mathrm{H}, \mathrm{CH}_{2} \mathrm{NCH}_{2}\right), 4.14(\mathrm{dd}, J=4.8,8.8 \mathrm{~Hz}$, 1H, H-3'), 3.93 (dd, $J=4.0,7.6 \mathrm{~Hz}, 1 \mathrm{H}, \mathrm{H}-4$ '), 3.65 (ddd, $J=4.4,9.2,11.9 \mathrm{~Hz}, 1 \mathrm{H}, \mathrm{H}-5$ 'a), 3.54 (ddd, $J=4.4,5.7$, $12.0 \mathrm{~Hz}, 1 \mathrm{H}, \mathrm{H}-5 \mathrm{~b}$ ), 3.07 (q, $J=7.3 \mathrm{~Hz}, 2 \mathrm{H}, \mathrm{SCH}_{2}$ ), $1.68 \sim 1.78\left(\mathrm{~m}, 2 \mathrm{H}, \mathrm{CH}_{2} \mathrm{CH}_{2} \mathrm{CH}_{2}\right), 1.59 \sim 1.65(\mathrm{~m}, 4 \mathrm{H}$, $\mathrm{CH}_{2} \mathrm{CH}_{2} \mathrm{CH}_{2}$ ), 1.33 (t, $\left.J=7.2 \mathrm{~Hz}, 3 \mathrm{H}, \mathrm{CH}_{3}\right) ;{ }^{13} \mathrm{C}$ NMR (DMSO- $\left.d_{6}, 100 \mathrm{MHz}\right) \delta: 163.0,152.4,151.3,137.4,117.0$, 87.3, 85.4, 79.1, 73.4, 70.4, 61.5, 45.7, 25.6, 24.7, 24.2, 22.4, 14.8; IR (KBr) v: 3347, 2930, 2854, 1577, 1444, 1343, 1316, 1249, 1218, 1118, $1074 \mathrm{~cm}^{-1}$; HRMS (ESI) calcd for $\mathrm{C}_{17} \mathrm{H}_{25} \mathrm{~N}_{5} \mathrm{O}_{4} \mathrm{~S} \quad[\mathrm{M}+\mathrm{H}]^{+}$396.1706, found 396.1699.

6-吗啡啉基-2-乙硫基嘌呤核苷(5h): 白色固体, 收 
率 69\%. m.p. 82 $84{ }^{\circ} \mathrm{C} ;{ }^{1} \mathrm{H}$ NMR (DMSO- $d_{6}, 400 \mathrm{MHz}$ ) $\delta: 8.30$ (s, 1H, H-8), 5.86 (d, $\left.J=5.8 \mathrm{~Hz}, 1 \mathrm{H}, \mathrm{H}-1^{\prime}\right), 5.44$ (d, $J=6.1 \mathrm{~Hz}, 1 \mathrm{H}, \mathrm{OH}), 5.19$ (d, $J=4.9 \mathrm{~Hz}, 1 \mathrm{H}, \mathrm{OH}), 5.04(\mathrm{t}$, $J=5.4 \mathrm{~Hz}, 1 \mathrm{H}, \mathrm{OH}), 4.56\left(\mathrm{dd}, J=5.6,11.2 \mathrm{~Hz}, 1 \mathrm{H}, \mathrm{H}-2^{\prime}\right)$, $4.20\left(\mathrm{~m}, 4 \mathrm{H}, \mathrm{CH}_{2} \mathrm{NCH}_{2}\right), 4.14(\mathrm{dd}, J=4.4,8.4 \mathrm{~Hz}, 1 \mathrm{H}$, H-3'), 3.93 (dd, $J=3.6,7.2 \mathrm{~Hz}, 1 \mathrm{H}, \mathrm{H}-4$ '), 3.72 (t, $J=4.4$ $\mathrm{Hz}, 4 \mathrm{H}, \mathrm{CH}_{2} \mathrm{OCH}_{2}$ ), 3.65 (ddd, $J=4.4,9.2,11.9 \mathrm{~Hz}, 1 \mathrm{H}$, H-5'a), 3.55 (ddd, $J=4.6,5.2,12.0 \mathrm{~Hz}, 1 \mathrm{H}, \mathrm{H}-5$ 'b), 3.08 (q, $J=7.1 \mathrm{~Hz}, 2 \mathrm{H}, \mathrm{SCH}_{2}$ ), $1.33\left(\mathrm{t}, J=7.3 \mathrm{~Hz}, 3 \mathrm{H}, \mathrm{CH}_{3}\right.$ ); ${ }^{13} \mathrm{C}$ NMR (DMSO- $\left.d_{6}, 100 \mathrm{MHz}\right) \delta: 163.1,152.5,151.4$, $137.9,117.2,87.3,85.4,79.1,73.3,70.3,66.1,61.4,45.2$, 24.7, 14.8; IR (KBr) v: 3338, 2928, 1616, 1583, 1477, 1451, 1340, 1298, 1242, 1111, $1079 \mathrm{~cm}^{-1}$; HRMS (ESI) calcd for $\mathrm{C}_{16} \mathrm{H}_{23} \mathrm{~N}_{5} \mathrm{O}_{5} \mathrm{~S}[\mathrm{M}+\mathrm{H}]^{+}$398.1499, found 398.1493 .

6-(4-甲基哌嗪-1-基)-2-乙硫基嘌呤核苷(5i): 白色 固体, 收率 48\%. m.p. 88 90 ${ }^{\circ} \mathrm{C} ;{ }^{1} \mathrm{H}$ NMR (DMSO- $d_{6}$, $400 \mathrm{MHz}) \delta: 8.28(\mathrm{~s}, 1 \mathrm{H}, \mathrm{H}-8), 5.86(\mathrm{~d}, J=5.8 \mathrm{~Hz}, 1 \mathrm{H}$, H-1'), 5.44 (d, $J=6.0 \mathrm{~Hz}, 1 \mathrm{H}, \mathrm{OH}), 5.19$ (d, $J=4.8 \mathrm{~Hz}$, $1 \mathrm{H}, \mathrm{OH}), 5.05$ (t, $J=5.4 \mathrm{~Hz}, 1 \mathrm{H}, \mathrm{OH}), 4.56$ (dd, $J=5.6$, $\left.10.8 \mathrm{~Hz}, 1 \mathrm{H}, \mathrm{H}-2^{\prime}\right), 4.20$ (m, 4H, $\mathrm{CH}_{2} \mathrm{NCH}_{2}$ ), 4.14 (dd, $J=$ 4.4, 8.4 Hz, 1H, H-3'), 3.93 (dd, J=3.6, 7.2 Hz, 1H, H-4'), 3.65 (ddd, $J=4.4,9.2,11.9 \mathrm{~Hz}, 1 \mathrm{H}, \mathrm{H}-5$ 'a), 3.54 (ddd, $J=$ 4.6, 5.1, $12.0 \mathrm{~Hz}, 1 \mathrm{H}, \mathrm{H}-5$ 'b), 3.07 (q, $J=7.1 \mathrm{~Hz}, 2 \mathrm{H}$, $\mathrm{SCH}_{2}$ ), 2.42 (t, $J=4.4 \mathrm{~Hz}, 4 \mathrm{H}, \mathrm{CH}_{2} \mathrm{NCH}_{3} \mathrm{CH}_{2}$ ), 2.22 (s, $3 \mathrm{H}, \mathrm{NCH}_{3}$ ), 1.33 (t, $J=7.2 \mathrm{~Hz}, 3 \mathrm{H}, \mathrm{SCH}_{2} \mathrm{CH}_{3}$ ); ${ }^{13} \mathrm{C} \mathrm{NMR}$ (DMSO- $\left.d_{6}, 100 \mathrm{MHz}\right) \delta: 163.1,152.4,151.4,137.7,117.1$, 87.3, 85.4, 79.2, 73.4, 70.4, 61.4, 54.5, 45.7, 44.3, 24.7, 14.8; IR (KBr) v: 3352, 2927, 2801, 1578, 1452, 1320, 1290, 1251, 1219, 1136, 1082, 1050, $1006 \mathrm{~cm}^{-1}$; HRMS (ESI) calcd for $\mathrm{C}_{17} \mathrm{H}_{26} \mathrm{~N}_{6} \mathrm{O}_{4} \mathrm{~S}[\mathrm{M}+\mathrm{H}]^{+}$411.1815, found 411.1810.

6-噻吩乙氨基-2-乙硫基嘌呤核苷 $(\mathbf{5 j})$ : 白色固体, 收率 76\%. m.p. $128 \sim 130{ }^{\circ} \mathrm{C} ;{ }^{1} \mathrm{H}$ NMR (DMSO- $d_{6}, 400$ MHz) $\delta: 8.24$ (s, 1H, H-8), 8.10 (br s, 1H, NH), 7.33 (dd, $J=0.9,5.0 \mathrm{~Hz}, 1 \mathrm{H}, \mathrm{SCHCH}), 6.96(\mathrm{dd}, J=3.4,4.9 \mathrm{~Hz}$, $1 \mathrm{H}, \mathrm{CHCHCH}), 6.92(\mathrm{~m}, 1 \mathrm{H}, \mathrm{CCHCH}), 5.83(\mathrm{~d}, J=5.9$
$\left.\mathrm{Hz}, 1 \mathrm{H}, \mathrm{H}-1^{\prime}\right), 5.44$ (d, $\left.J=6.0 \mathrm{~Hz}, 1 \mathrm{H}, \mathrm{OH}\right), 5.19$ (d, $J=$ $4.7 \mathrm{~Hz}, 1 \mathrm{H}, \mathrm{OH}), 5.09 \sim 5.13(\mathrm{~m}, 1 \mathrm{H}, \mathrm{OH}), 4.61(\mathrm{dd}, J=$ 5.7, $\left.11.0 \mathrm{~Hz}, 1 \mathrm{H}, \mathrm{H}-2^{\prime}\right), 4.15$ (dd, $J=4.4,8.0 \mathrm{~Hz}, 1 \mathrm{H}$, H-3'), $3.94 \sim 4.07\left(\mathrm{~m}, 1 \mathrm{H}, \mathrm{H}-4^{\prime}\right), 3.72 \sim 3.81(\mathrm{~m}, 2 \mathrm{H}$, $\mathrm{NHCH}_{2}$ ), 3.66 (ddd, $J=4.3,8.9,11.6 \mathrm{~Hz}, 1 \mathrm{H}, \mathrm{H}-5$ 'a), 3.55 (ddd, $J=4.3,5.9,11.6 \mathrm{~Hz}, 1 \mathrm{H}, \mathrm{H}-5$ 'b), $3.15 \sim 3.33(\mathrm{~m}, 2 \mathrm{H}$, $\mathrm{SCH}_{2}$ ), $3.08 \sim 3.13\left(\mathrm{~m}, 2 \mathrm{H}, \mathrm{NHCH}_{2} \mathrm{CH}_{2}\right), 1.35$ (t, $J=7.2$ $\mathrm{Hz}, 3 \mathrm{H}, \mathrm{CH}_{3}$ ); ${ }^{13} \mathrm{C}$ NMR (DMSO- $\left.d_{6}, 100 \mathrm{MHz}\right) \delta: 163.6$, $153.7,149.5,141.4,138.7,126.9,125.1,124.0,117.3$, 87.4, 85.5, 79.2, 73.3, 70.5, 61.6, 41.6, 29.2, 24.8, 15.1; IR (KBr) $v$ : 3356, 2926, 1617, 1583, 1476, 1338, 1300, 1113, $1078 \mathrm{~cm}^{-1}$; HRMS (ESI) calcd for $\mathrm{C}_{18} \mathrm{H}_{23} \mathrm{~N}_{5} \mathrm{O}_{4} \mathrm{~S}_{2}[\mathrm{M}+\mathrm{H}]^{+}$ 438.1270, found 438.1265.

辅助材料(Supporting Information) 目标化合物的核 磁、红外和高分辨质谱谱图. 这些材料可以免费从本刊 网站(http://sioc-journal.cn/)上下载.

\section{References}

[1] Pravin, P.; Abhay, N.; Krishnachaya, G. A. J. Org. Chem. 2014, 79(5), 2331.

[2] Siyang, H. X.; Wu, X. R.; Liu, H. L.; Wu, X. Y.; Liu, P. N. J. Org. Chem. 2014, 79(3), 1505.

[3] Xia, R.; Xie, M. S.; Niu, H. Y.; Qu, G. R.; Guo, H. M. Org. Lett. 2014, 16(2), 444.

[4] Giulia, V.; Martina, M.; Deborah, S.; Emmanuele, C.; Miroslava, K.; Giovanni, M.; Silvia, A.; Marco, R.; Maurizio, B. ACS Comb. Sci. 2014, 16(4), 168.

[5] Katrin, M.; Klaus, R. Ach. Pharm. Chem. Life Sci. 2006, 339, 115.

[6] Wang, T.; Du, H. G. Chin. J. Org. Chem. 2012, 32, 1720 (in Chinese). (汪蹈, 杜洪光, 有机化学, 2012, 32, 1720.)

[7] He, Q. W.; Du, H. G. Chin. J. Org. Chem. 2012, 32, 1678 (in Chinese). (何琦文, 杜洪光, 有机化学, 2012, 32, 1678.)

[8] Deng, C. E.; Li, S. L.; Liu, X W.; Du, H. G. Chin. J. Org. Chem. 2013, 33, 1741 (in Chinese). (邓聪迩, 李顺来, 刘祥伟, 杜洪光, 有机化学, 2013, 33, 1741.)

[9] Liu, G. C.; Xu, J. X.; Park, K. C.; Chen, N.; Zhang, S.; Ding, Z. R.; Wang, F.; Du, H. G. Tetrahedron 2011, 67, 5156.

[10] Wu, Z. J.; Li, S. L.; Ding, Z. R.; Du, H. G. Chin. J. Org. Chem. 2011, 31, 1618 (in Chinese).

(吴兆军, 李顺来, 丁忠仁, 杜洪光, 有机化学, 2011, 31, 1618.) 